\title{
Occupation-Related Biological Health Hazards and Infection Control Practices among Indian Veterinarians
}

\author{
Rajendra Palkhade $\mathbb{D}^{1}{ }^{1}$ SukhDev Mishra $\mathbb{D}^{1},{ }^{1}$ and Sukhadeo Barbuddhe ${ }^{2}$ \\ ${ }^{1}$ ICMR-National Institute of Occupational Health, Meghaninagar, Ahmedabad, Gujarat, India \\ ${ }^{2}$ ICAR-National Research Centre on Meat, Chenicherla, Hyderabad, India \\ Correspondence should be addressed to Rajendra Palkhade; drpalkhade@gmail.com
}

Received 3 September 2021; Revised 23 December 2021; Accepted 3 February 2022; Published 24 February 2022

Academic Editor: Antonio Ortega-Pacheco

Copyright ( 2022 Rajendra Palkhade et al. This is an open access article distributed under the Creative Commons Attribution License, which permits unrestricted use, distribution, and reproduction in any medium, provided the original work is properly cited.

\begin{abstract}
Veterinarians experience different types of health hazards from their occupation. Studies on the prevalence and occurrence of biological health hazards in veterinary medicine in India are scant and probably underreported. Thus, we sought to assess the biological health hazards and infection control practices (ICPs) among veterinarians from the states of Gujarat and Maharashtra, India. A cross-sectional survey was conducted among veterinarians $(n=562)$ from Gujarat and Maharashtra states in India to identify biological health hazards and ICPs for the prevention of occupational health hazards during 2016-2017 by personally contacting them. Responses regarding a biological hazard and ICPs were recorded. Descriptive analysis was attempted, and continuous variables are presented as the mean \pm SD. Categorical variables are reported as counts and percentages (\%). Most of the veterinarians (49.3\%) worked in the field and were continuously exposed to different types of biological health hazards, especially zoonoses, ranging from mild and self-limiting to fatal diseases (e.g., brucellosis (subclinical and clinical form) and rabies (fatal)) without common prophylactic vaccinations, such as rabies and tetanus. While inquiring medical health status of the veterinarians, only $35.8 \%$ of the total respondents underwent a routine medical health checkup within the past year, and $56.9 \%$ did not receive a routine dose of an anthelmintic for deworming. Forty-nine percent of the respondents took all necessary precautions, including wearing an apron, facemask, and gloves. In contrast, $10.2 \%$ of the respondents wore only an apron, and $8.4 \%$ of respondents did not take any precautions while performing their day-to-day work. In total, $40.2 \%$ of the respondents followed the proper method of handwashing, that is, washing hands between patient examinations. In contrast, $27.9 \%$ of the respondents washed their hands once after completing the work. The majority of the respondents (87.7\%) reported an urgent need for occupational hazards and safety (OHS) training in continued veterinary education (CVE) programs. The present study demonstrates that veterinarians in the states of Gujarat and Maharashtra in India pay less attention to their own health that may increase the risk of occupation-related biological health hazards. These results suggest that safety and ICPs are not prioritized, which are serious concerns. These findings may be useful for developing policies to prevent occupationally related biological health hazards among veterinarians in India.
\end{abstract}

\section{Introduction}

Veterinarians play a crucial role in the development, wellbeing, health care, and welfare of animals and in upgrading the rural economy of India by empowering socioeconomic classes. The main components of the job profile of veterinarians in India include prevention, treatment, management of animal diseases, improvement of production, and extension activities to implement government schemes to elevate the socioeconomic class through animal husbandry activities. Most farmers in rural areas receive veterinary health care services from government-appointed veterinarians [1]. Veterinarians also contribute to public health and protect humans against zoonotic diseases by using their scientific knowledge and technical skills. While performing their day-to-day work, veterinarians come in close contact with various sick animals and birds, and some animals are carriers of infections despite appearing healthy. Such apparently healthy animals also pose a risk of transmitting various zoonotic diseases to veterinarians [2]. Zoonoses are 
diseases and infections that are naturally transmitted between vertebrate animals and humans [3]. Due to the virtue of their occupation, veterinarians are exposed to different occupational health hazards, viz., physical, chemical, biological $[4,5]$, and psychological hazards. Veterinarians are exposed to possible carcinogens, including radiation, pesticides, anesthetic, and zoonotic infections, including viruses [6]. Occupational health and safety (OHS) is often focused on workers involved in manufacturing and construction, but infectious diseases often more frequently occur in occupational settings [7].

Veterinarians are at high risk for occupation-related biological health hazards, especially in countries that exercise traditional farming activities, where keeping backyard livestock remains customary, and there is low knowledge of zoonoses among livestock keepers [8].

Veterinarians in Australia are considered a high-risk group for occupational hazards [4]. Approximately 75\% of emerging human infectious diseases are directly or indirectly linked to animal origins [9]. This finding highlights the importance of identifying occupational health hazards among veterinarians.

The most commonly reported zoonotic diseases in veterinarians include ringworm $[10,11]$; cat scratch fever, which is also known as Bartonellosis and caused by Bartoneolla henselae; salmonellosis $[10,12]$; brucellosis $[13,14]$; leptospirosis [15]; and Q-fever [16, 17]. Despite the risk of different zoonotic diseases in the field of veterinary medicine, a recent study conducted among United Arab Emirates' large-animal (39\%) and small-animal veterinarians (41\%) showed a failure to use personal protective equipment (PPE) while handling blood samples [18].

Complete elimination of the potential of contracting zoonotic diseases is an unrealistic goal; thus, the focus should be on preventing diseases by decreasing the risk. New activities and advanced technologies have generated new zoonotic and occupational risks. The Indian subcontinent is one of the four global hotspots at increased risk for the emergence of new infectious diseases [19]. Therefore, the identification/evaluation of biological hazards among Indian veterinarians is extremely important.

The risk of spreading zoonotic infections has always represented a threat to public health in terms of productive and economic losses, and veterinarians may act as carriers. To the best of our knowledge, comprehensive studies have not been conducted to date to identify occupation-related biological health hazards and ICPs among Indian veterinarians. There is an urgent need to assess occupational hazards in veterinary practice to develop strategies for prevention. Hence, the objectives of the present survey was to assess biological health hazards and ICPs among veterinarians in the states of Gujarat and Maharashtra, India, which can help to encourage science-based biological health hazard prevention policies for veterinarians and associated workers.

\section{Materials and Methods}

This study was conducted among veterinarians $(n=562)$ in the states of Gujarat and Maharashtra, India. The survey tool (a set of questionnaires) was designed by reviewing the internationally conducted studies [10, 20, 21] and [22] on related topics of survey objectives. Experts in this profession were consulted to identify different occupation-related biological health hazards and ICPs. The questionnaire was modified thereafter on the feedback received from the pretest conducted among veterinarians, which was not included in the survey analysis. Veterinarians were included in the study if they fulfilled the inclusion criteria and were willing to participate. During 2016-2017, the self-assessed questionnaire was completed by veterinarians who were personally contacted. Prior to the inclusion of individual veterinarians in the study, the purpose of the study was described to the veterinarians, and written consent was obtained. Only veterinarians with recent veterinary work experience for at least 2 years and those who were continuously working despite being retired from service were included in the study. Retired (nonpracticing) veterinarians, veterinarians with less than 2 years of the experienced, nonclinical academic staff of veterinary colleges/universities, and veterinarians involved in administrative/extension activities were excluded from the study. All questions were of the close-ended type, and the participants were asked to rank or select from a list of provided options.

Recall for each response in the present study was limited to occurrences within the last 2 years. Data were collected on biological health hazards and ICPs based on the following categories:

(1) Individual health: prophylactic vaccination, routine medical health checkup, screening for zoonotic diseases, deworming doses, any ailments/allergy, and medical treatment sought at the time of the interview.

(2) Public health: sharing and discussion of zoonosis among subordinates, clients, farmers, general population, and physicians.

(3) ICPs: handwashing pattern, use of PPE, awareness about different types of occupational health hazards (physical, chemical, and biological hazards and allergies) in the field of veterinary medicine, and source(s) of their information about occupational hazards and risk.

For sample size estimation, we assumed $p=0.05$ with the desired precision of $5 \%$ and a confidence level of $95 \%$ as suggested by [23] in case of unavailability of suitable evidence [23].

The sample size was calculated using the formula $n \geq p(1-p) *(Z / d)^{2}$, where $d$ is the desired precision. The study required a minimum sample size of 427 subjects considering a $10 \%$ nonresponse rate; however, to increase the precision of the study results, we were able to collect and analyze the responses from 562 subjects on the study questionnaire.

The study was approved by the Institutional Human Ethical Committee at ICMR-National Institute of Occupational Health, Ahmedabad (Agenda No. 3.6/2015).

All recorded questionnaire data were transcribed into customized databases using data entry screens in the EpiInfo 7 software. The data were doubly entered to minimize 
any entry errors. All data errors were reconciled by referring to paper-based data entry. Continuous variables, such as age, are presented as the mean $\pm \mathrm{SD}$, and all other attributes of a categorical nature are reported as counts $(n)$ and percentages (\%). The 95\% confidence interval was performed for reported data. All the statistical analysis was performed using $R$ Software version 4 .

\section{Results}

The results illustrated that the mean age of the veterinarians working in Gujarat and Maharashtra state was $39.94 \pm 9.75$ years, and most of them worked for more than 50 hours a week.

The majority $(80.9 \%)$ of the veterinarians agreed that they have suffered needlestick injuries in the last 2 years. Needlestick injuries are related to physical hazards, such as local inflammation, and subsequent biological hazards. Hence, the material present in the needle at the time of the puncture event was assessed. Most respondents (60.1\%, $28.5 \%$, and $3.4 \%$ ) reported that needles contained nonspecific drugs, antibiotics, and vaccines (rabies and brucella vaccine), respectively, at the time of the needle puncture event (Table 1).

Data were collected on individual health. Only $35.8 \%$ of the total respondents had undergone a routine medical health checkup within the past year, and $36.4 \%$ of respondents had never undergone a routine medical health checkup. Despite being a high-risk occupational group and constantly being surrounded by animals with increased chances of parasitic infestation, approximately $56.9 \%$ of the respondents did not receive a routine dose of an anthelmintic for routine deworming. In contrast, $18.9 \%, 12.5 \%$, and $11.8 \%$ (total $43.2 \%$ ) of the respondents received an anthelmintic dose within 12,6 , and 4 months, respectively. Among the respondents, only $27.0 \%$ underwent screening for zoonotic infections. Total of eight individuals were found positive for brucellosis (for brucellosis screening, different tests were mentioned by the respondents, such as Rose Bengal plate test (RBPT), IgM, and IgG antibiotics detection through ELISA), and one individual was positive for ringworm infections. Half of the respondents $(49.1 \%)$ did not report any ailments at the time of the study, whereas the remaining respondents suffered from different ailments such as anxiety (9.5\%), diabetes (4.7\%), enteric disorders (6.4\%), hypotension or hypertension (14.9\%), and allergies (10.4\%). Regarding allergies, the veterinarians suffered from different types of allergies such as fur/dandruff (13.1\%), latex gloves $(1.6 \%)$, chemicals/drugs (4.9\%), and vaginal secretions (1.6\%). Moreover, $12.5 \%$ of the respondents suffered from allergies so severe that they sought medical attention. Half of the surveyed population $(49.3 \%)$ did not receive any prophylactic vaccinations, such as rabies and tetanus. A total of $39.5 \%$ and $11.1 \%$ of the respondents received rabies and tetanus vaccines, respectively (Table 2 ).

Regarding ICPs, approximately half of the respondents $(49.0 \%)$ were taking all necessary precautions while dealing with animals, including wearing aprons, facemasks, and gloves. In contrast, $10.2 \%$ of the respondents wore only an
TABLE 1: Needle-stick-related information among Indian veterinarians.

\begin{tabular}{lcc}
\hline Description & $n(\%)$ & $95 \%$ CI \\
\hline Needlestick injury $(n=562)$ & & \\
Yes & $455(80.9)$ & $(77.49,83.99)$ \\
No & $107(19.0)$ & $(16.00,22.50)$ \\
Material present at the time of puncture event & $(n=536)$ \\
Brucella vaccine & $3(0.6)$ & $(0.1,1.74)$ \\
Rabies vaccine & $15(2.8)$ & $(1.6,4.63)$ \\
Nonspecific drug & $322(60.1)$ & $(55.8,64.14)$ \\
Antibiotics & $153(28.5)$ & $(24.8,32.52)$ \\
Anthelmintic & $2(0.4)$ & $(0.1,1.46)$ \\
Steroids & $8(1.5)$ & $(0.7,2.99)$ \\
Not applicable & $33(6.2)$ & $(4.4,8.56)$ \\
\hline
\end{tabular}

apron, and $8.4 \%$ of the respondents routinely worked in the field without taking any precautions. Regarding handwashing, $40.2 \%$ of the respondents followed proper handwashing, that is, washing hands between patient examinations, whereas $27.9 \%$ of the respondents only washed their hands once after finishing their work day. The practice of washing hands before and after work was followed by $11.4 \%$ of respondents and $6.2 \%$ of the respondents only washed their hands before eating (Table 3 ).

Regarding professional collaboration, $70.2 \%$ and $23.2 \%$ of the respondents were never and rarely contacted by physicians, respectively. Moreover, $55.6 \%$ and $36.5 \%$ of the respondents never or rarely contacted physicians, respectively, regarding zoonotic diseases (Table 4).

Regarding knowledge about OHS, approximately 35\% (34.9\%) and $19.9 \%$ of the total respondents interviewed were aware of physical and biological health hazards in the veterinary profession, whereas $3.1 \%$ and $3.6 \%$ were aware of chemical hazards and allergies, respectively. Meetings/ training $(38.8 \%)$ followed by the Internet (39.9\%), news bulletins (14.2\%), and conferences (7.1\%) were reported as tools that played an important role in keeping veterinarians updated about OHS. Most of the respondents (87.7\%) expressed an urgent need for the inclusion of OHS training in continuing veterinary education (CVE) programs. Half of the respondents (56.6\%) discussed zoonosis occasionally, $6.5 \%$ weekly, $13.6 \%$ daily, and $12.6 \%$ several times a day among their subordinates and client population (Table 5).

\section{Discussion}

There are three main important issues related to occupational health and management: individual health, workplace culture (ICPs), and physical issues. In this study, we focused on the individual health and workplace culture among veterinary medicine professionals in the states of Gujarat and Maharashtra, India, to explore the biological health hazards related to this occupation and the ICPs adopted by the veterinarians in the study area in their day-to-day work. The present study revealed that most of the veterinarians worked for more than 50 hours a week, which is similar to that reported for Australian zoo veterinarians [24]. Despite awareness of issues, such as mental and physical stresses [25], awareness of biological health hazards, including 
TABLE 2: Status of medical health checkups among Indian veterinarians.

\begin{tabular}{|c|c|c|}
\hline Description & $n(\%)$ & $95 \% \mathrm{CI}$ \\
\hline \multicolumn{3}{|c|}{$\begin{array}{l}\text { Time when last routine medical health checkup was performed } \\
(n=558)\end{array}$} \\
\hline One month & $10(1.8)$ & $(0.9,3.34)$ \\
\hline 1 year & $190(34.0)$ & $(30.2$, \\
\hline 2 years & $78(14)$ & $(11.3$, \\
\hline $3-5 y$ & $77(1$ & $(11$. \\
\hline No medical checkup & $203(36$ & (32. \\
\hline \multicolumn{3}{|c|}{ Ailments at time of the interview $(n=550)$} \\
\hline Anxiety & $52(9.5)$ & $(7.2,12.22)$ \\
\hline Allergies & $57(10.4)$ & $(8,13.22)$ \\
\hline Diabetes & $26(4.7)$ & $(3.2,6.88)$ \\
\hline Enteric disorder & $35(6.4)$ & $(4.5,8.76)$ \\
\hline d hypertension & $82(14.9)$ & $(12.1,18.15)$ \\
\hline Hypercholesterolemia & $9(1.6)$ & $(0.1,3.15)$ \\
\hline No ailments & $270(49.1)$ & $(44.9,53.27)$ \\
\hline Other & $19(3.5)$ & $(2.2,5.39)$ \\
\hline
\end{tabular}

Allergies at time of the interview $(n=550)$

$\begin{array}{lcc}\text { Fur/dandruff } & 72(13.1) & (10,16.2) \\ \text { Latex gloves } & 9(1.6) & (0.8,3.15) \\ \text { Chemicals/drugs } & 27(4.9) & (3.3,7.09) \\ \text { Vaginal secretion } & 9(1.6) & (0.8,3.15) \\ \text { Amniotic fluid } & 6(1.1) & (0.4,2.44) \\ \text { Any other } & 8(1.5) & (0.6,2.92) \\ \text { No allergy } & 419(76.2) & (72.4,79.55)\end{array}$

Sought medical attention for your allergy ailments during the last 2 years $(n=553)$

\begin{tabular}{lcc} 
Yes & $69(12.5)$ & $(9.9,15.52)$ \\
No & $257(46.5)$ & $(42.3,50.65)$ \\
Not required & $175(31.6)$ & $(27.9,35.65)$ \\
Do not remember & $52(9.4)$ & $(7.2,12.16)$ \\
4 months & $66(11.8)$ & $(9.3,14.72)$ \\
6 months & $70(12.5)$ & $(9.9,15.5)$ \\
1 year & $106(18.9)$ & $(15.8,22.36)$ \\
No routine deworming & $319(56.9)$ & $(52.7,60.9)$ \\
\hline
\end{tabular}

Screening for any zoonotic infection during the last

2 years. $(n=562)$

\begin{tabular}{lcc} 
Yes & $152(27.0)$ & $(23.4,30.77)$ \\
No & $337(59.8)$ & $(55.6,63.72)$ \\
Facility not available & $67(11.9)$ & $(9.4,14.84)$ \\
Not interested & $6(1.3)$ & $(0.6,2.85)$ \\
\hline
\end{tabular}

If yes, then specify the zoonotic diseases $(n=552)$

\begin{tabular}{lcc} 
Brucellosis $^{*}$ & $148(26.8)$ & $(23,30.67)$ \\
Toxoplasmosis $^{\#}$ & $1(0.2)$ & - \\
Leishmaniasis $^{\#}$ & $1(0.2)$ & - \\
Ringworm (dermatophytosis) $^{\#}$ & $1(0.2)$ & - \\
Tuberculosis $^{\#}$ & $1(0.2)$ & - \\
Not applicable & $400(72.5)$ & $(68.5,76.03)$ \\
\hline
\end{tabular}

Status of your prophylactic vaccination during the past 2 years $(n=560)$

\begin{tabular}{lcc} 
Rabies & $221(39.5)$ & $(35.5,43.58)$ \\
Tetanus & $62(11.1)$ & $(8.7,13.97)$ \\
Hepatitis A/B & $1(0.2)$ & - \\
No vaccine received & $276(49.3)$ & $(45.1,53.42)$ \\
\hline
\end{tabular}

${ }^{\#} \mathrm{CI}$ not calculated due to insufficient $n$.

mycotic infections, mange, swine erysipelas, anthrax and tuberculosis, brucellosis [13, 14, 26], leptospirosis [15, 27], Q-fever [16, 17], and Toxoplasma gondii (due to its
TABLE 3: Infection control practices (ICPs) among Indian veterinarians.

\begin{tabular}{lcc}
\hline Description & $n(\%)$ & $95 \%$ CI \\
\hline Type of precaution followed to avoid $z$ zoonotic hazards $(n=561)$ \\
No precaution & $47(8.4)$ & $(6.3,11)$ \\
Apron only & $57(10.2)$ & $(7.9,12.97)$ \\
Gloves only & $182(32.4)$ & $(28.7,36.44)$ \\
Apron, facemask, and gloves & $275(49.0)$ & $(44.9,53.15)$ \\
Method of handwashing with soap & and disinfectant & $(n=562)$ \\
Between patient examinations & $226(40.2)$ & $(36.2,44.33)$ \\
Once after completing work & $157(27.9)$ & $(24.3,31.8)$ \\
Seldom & $10(1.8)$ & $(0.9,3.31)$ \\
Never & $1(0.2)$ & - \\
Before eating only & $35(6.2)$ & $(4.5,8.58)$ \\
Frequently & $69(12.3)$ & $(9.8,15.28)$ \\
Before and after work & $64(11.4)$ & $(9,14.31)$ \\
\hline
\end{tabular}

${ }^{\#} \mathrm{CI}$ not calculated due to insufficient $n$.

TABLE 4: Interprofessional collaboration to prevent zoonoses among Indian veterinarians and physicians.

\begin{tabular}{|c|c|c|}
\hline Description & $n(\%)$ & $95 \% \mathrm{CI}$ \\
\hline \multicolumn{3}{|c|}{$\begin{array}{l}\text { Physicians (human) consulted you for advice on zoonotic diseases } \\
(n=560)\end{array}$} \\
\hline Several times/week & $15(2.7)$ & $(1.6,4.43)$ \\
\hline Several times/month & $22(3.9)$ & $(2.5,5.93)$ \\
\hline Rarely & $130(23.2)$ & $(19.9,26.9)$ \\
\hline Never & $393(70.2)$ & $(66.2,73.82)$ \\
\hline Several times/week & $8(1.4)$ & $(0.6,2.89)$ \\
\hline Several times/month & $10(1.8)$ & $(0.9,3.35)$ \\
\hline Several times/year & $26(4.7)$ & $(3.1,6.81)$ \\
\hline Rarely & $203(36.5)$ & $(32.6,40.6)$ \\
\hline Never & $309(55.6)$ & $(51.4,59.66)$ \\
\hline
\end{tabular}

teratogenic potential among working pregnant female veterinarians, if infected in the first trimester) [28], remains one of the prominent risks in this occupation. Furthermore, female veterinarians should be cautious before planning for pregnancy and throughout their pregnancy regarding the possibility of zoonosis infection while working in the field.

4.1. Individual Health. Animals are often uncooperative; hence, veterinarians are at higher risk of needlestick injury than other health care workers. Occupational needlestick injuries may cause transmission of infectious agents, which represents another major public health concern. The prevalence of needlestick injury (sharp management) among veterinarians in the same study area was previously found to be very high $(80.9 \%)$ [5], and various studies have shown that it is the most common source of injury among these professionals. Transmission of infectious agents and other harmful agents through needlesticks during work hours is a serious public health concern [29]. In the present study, $28.5 \%$ and $3.4 \%$ of respondents mentioned needles containing antibiotics and vaccines (brucella and rabies vaccines), respectively, at the time of the puncture event. Similar findings were reported in a study conducted among veterinarians in Illinois [20], that is, needles contained antibiotics, vaccines, anesthetics, and animal blood at the time of the puncture event. A study 
TABLE 5: Knowledge about occupational health and safety (OHS) among Indian veterinarians.

\begin{tabular}{lcc}
\hline Description & $n(\%)$ & $95 \% \mathrm{CI}$ \\
\hline Awareness about different occupational hazards & in veterinary \\
medicine $(n=478)$ & \\
Physical hazards & $167(34.9)$ & $(30.8,39.33)$ \\
Biological hazards & $95(19.9)$ & $(16.5,23.71)$ \\
Chemical hazards & $15(3.1)$ & $(1.8,5.18)$ \\
Allergies & $17(3.6)$ & $(2.2,5.68)$ \\
Psychological/mental & $3(0.6)$ & $(0.1,1.95)$ \\
All of the above & $165(34.5)$ & $(30.3,38.9)$ \\
None of the above & $16(3.3)$ & $(2,5.43)$ \\
Source of information for occupational hazards $(n=551)$ \\
News bulletin & $78(14.2)$ & $(11.4,17.34)$ \\
Internet & $220(39.9)$ & $(35.9,44.08)$ \\
Conference & $39(7.1)$ & $(5.2,9.57)$ \\
Meeting/training & $214(38.8)$ & $(34.8,42.98)$ \\
Urgent need to include occupational hazards in CVE program \\
(n=554) & & \\
Yes & $486(87.7)$ & $(84.7,90.21)$ \\
No & $20(3.6)$ & $(2,5.56)$ \\
Cannot say & $27(4.9)$ & $(3.3,7.04)$ \\
Do not know & $21(3.8)$ & $(2.4,5.78)$ \\
Discussions among your client population/subordinates about \\
zoonotic diseases $(n=557)$ & & \\
Several times/day & $70(12.6)$ & $(10,15.61)$ \\
Daily & $76(13.6)$ & $(11,16.77)$ \\
Weekly & $36(6.5)$ & $(4.6,8.86)$ \\
Occasionally & $315(56.6)$ & $(52.4,60.61)$ \\
Never & $60(10.8)$ & $(8.4,13.65)$ \\
\hline
\end{tabular}

conducted among American female veterinarians $(n=457)$ reported that $6 \%$ and $3 \%$ experienced accidental exposure to rabies and Brucella abortus vaccine, respectively [30], which is similar to the findings of the present study. A comparatively increased incidence of accidental exposure to vaccines due to needlestick injury was reported among Canadian veterinarians $(26 \%(214 / 810))$ [10]. This stark difference may be due to differences in animal practices. Animal vaccines are not tested for safety in humans, so their adverse effects remain unexplored, which may affect the health of veterinarians.

4.1.1. Routine Medical Health Checkup. Despite routine handling of diseased and potentially infectious animals, infectious material, and work-related hazards, such as mental stress, $36.4 \%$ of the respondents did not undergo a routine medical health checkup in the past. In contrast, $35.8 \%$ of the respondents underwent a routine medical health checkup during the past 1 year, which is lower than the value in a study conducted among veterinarians from Western countries, where $87 \%$ consulted physicians within the previous 30 months [20]. This difference may be explained by the awareness of occupational hazards and the need for health checkups in Western countries as a job profile.

4.1.2. Ailments at the Time of Interview. At the time of the interview, half of the veterinarians had no ailments, whereas the remaining half of the respondents suffered from different ailments, such as anxiety (9.5\%), diabetes (4.7\%), enteric disorders (6.4\%), and hypotension or hypertension (14.9\%). Veterinarians and associated staff may be at higher risk for allergen exposure from both the animals (fur, dandruff, urine, etc.) that they treat and some of the therapeutic agents that they use in their daily practice [4]. Among the half of the respondents who suffered from ailments, $10.4 \%$ suffered from allergies, that is, fur/dandruff (13.1\%), latex gloves (1.6\%), chemicals/drug (4.9\%), vaginal secretion (1.6\%), and amniotic fluid (1.1\%). Allergies to body fluids, hair, dander, latex, and chemical allergens were also reported among Canadian veterinarians [10]. Dust and animal dung represent known allergic health hazards, whereas allergic rhinitis $[31,32]$ and conjunctivitis were the most commonly reported allergic conditions in animal-related occupations [33]. In the present study, no individual reported allergies due to antimicrobials, whereas Lessenger [20] reported that $12 \%$ of veterinarians suffered from the specific allergy. However, $12.5 \%$ of the respondents' allergies were so severe that they sought medical advice to control their allergies. The prevalence of allergies increased with the length of occupational exposure, and female veterinarians were more likely to develop allergies than male veterinarians $[10,11]$.

Despite the high risk for zoonotic infection, only $27 \%$ of the veterinarians were cautious about screening for zoonotic diseases. The majority (59.8\%) of the respondents did not undergo zoonotic disease screening in the past two years. Our findings are similar to those in a study conducted in the Northern part of the country (Punjab state) for brucellosis exposure among animal handlers, para veterinarians, and veterinarians, which reported that amongst the different tehsils (subdistricts), the veterinarians from Khanna were least likely to have been tested for brucellosis [34]. Screening of zoonotic diseases is very important for the health of the veterinarians; in a survey conducted among Australian veterinarians, wherein $44.9 \%(n=344)$ reported acquiring a zoonotic disease throughout their career [35], a higher percentage of positive for zoonoses reported among Australian veterinarians may be because of a higher percentage of screening of zoonotic diseases due to more awareness and availability of zoonotic diseases screening facility. A survey conducted among researchers in a veterinary facility in Nigeria reported a current knowledge of laboratory biosecurity and biosafety is limited [36]. This report is consistent with the finding that humans were exposed to biological hazards in this occupational setting largely due to known or unknown exposure to live animals or animal products. In particular, farm animal veterinarians (large animal veterinarians) are threefold more likely to contract zoonotic diseases than those working in other veterinary practices [20].

4.1.3. Prophylactic Vaccination. If ignored, bite injuries from animals may cause fatal diseases [11], such as rabies. Rabies is endemic in India, and India is a leading country of rabies-associated deaths. The incidence of human rabies in India is 20,565 per year, and dog bites (96.2\%) are the main cause [37]. However, half (49.3\%) of the veterinarians in the 
present study population did not receive common vaccinations, such as rabies and tetanus. Potentially due to increased awareness among wildlife veterinarians, $62.9 \%$ of these veterinarians had received prophylactic vaccination for rabies in the country [38]. Three doses for pre-exposure prophylaxis for rabies at days 0,7 , and 21 or 28 are recommended for veterinarians and animal handlers in India [39]. A study conducted among US veterinarians in Oregon found that $13.9 \%$ had never been vaccinated for rabies [40], and a lower percentage of prophylactic vaccination might be due to awareness about zoonoses among veterinarians from developed countries than developing countries. Despite the high risk of fatal zoonotic diseases among these professionals, internationally conducted studies among veterinarians have demonstrated a low rate of rabies immunization. For example, half $(47 \%)$ of Illinois veterinarians received a tetanus vaccine in the last 18 months, and other studies have reported a low rate of rabies immunization despite bite injuries [20].

\subsection{Infection Control Practices (ICPs)}

4.2.1. Use of Personal Protective Equipment (PPE). e USA [21], 18 of 71 (25.4\%) human infections occurred among veterinary personnel. The contraction of zoonoses by veterinarians and associated staff, for example, highlights the need for ICPs in veterinary medicine such as using PPE while handling animals to significantly minimize the risk of contracting zoonosis. In the present study, half $(49.0 \%)$ of the respondents wore aprons, facemasks, and gloves as a precaution to avoid zoonoses, which is less than the Australian veterinarians $(75.0 \%)$ as they used "adequate" PPE to avoid zoonoses [35]. However, $32.4 \%$ of the respondents only wore gloves, and $8.4 \%$ of the respondents worked in the field without PPE. Despite the high risk of contracting zoonotic diseases from wild animals, a comparatively increased percentage (16.7\%) of Indian wildlife veterinarians $(n=54)$ reported working in the field without taking any precautions or using PPE [38]. The incidences of fatal diseases, such as leptospirosis and bird flu, are reduced when proper PPE is used [15]. The higher percentage of respondents taking precautions in the present study may be due to increasing awareness among the veterinarians about biological hazards/zoonotic diseases. Similarly, Wright et al. [21] has also reported that PPE could prevent zoonoses to some extent among large-animal veterinarians in the USA [21]. We did not collect information on ICPs based on clinical cases. However, a study conducted among Australian veterinarians revealed that $60-70 \%$ did not use PPE while treating respiratory and neurological cases, whereas $40-50 \%$ of the respondents did not use PPE while treating gastrointestinal and dermatological cases [35]. A study focused exclusively on risk factors for Brucella infection among veterinarians showed a need for investigation to clarify the effectiveness of PPE to reduce Brucella infection [14]. A study also confirmed that brucellosis was found a common occupational zoonosis among veterinary personnel $(n=279)$.
4.2.2. Personal Hygiene. Personal hygiene is an important measure for reducing the risk of zoonosis and other diseases along with early recognition of infected animals, proper animal handling, and basic biosecurity precautions. Handto-hand contact has been reported as the most common form of disease transmission, so consistent hand hygiene is very important in reducing the risk of disease spread [41]. Veterinarians are at risk of methicillin-resistant Staphylococcus aureus (MRSA) infection [42], and handwashing can prevent such infections. Unwashed hands may pose a risk of nosocomial transmission among different patient species [21]. Proper handwashing before and after animal contacts and handling blood, body fluids, secretions, excretions, and equipment or articles is a significant tool to reduce the number of transient organisms. In the present study, $40.2 \%$ and $27.9 \%$ of the respondents washed their hands between patients and once after finishing work, respectively. Veterinarians from the USA reported lower rates of washing their hands between patient contacts (18\%) compared with the present study population [21]. In one UK study, handwashing practices by doctors were less common $(8.1 \%$ and $51.4 \%$ ) before and after patient contacts than those by nurses $(20.4 \%$ and $60.1 \%)$ [43]. Wearing gloves reduces the transmission of organisms by providing barrier protection but is not a substitute for hand hygiene [44].

\subsection{Interprofessional Collaboration}

4.3.1. Communication between Physicians and Veterinarians. Considering the ideology of "One Health," data were obtained to understand communication between veterinarians and physicians. Surprisingly, in the present survey, the majority $(70.2 \%)$ of respondents reported a lack of communication and mentioned that physicians never contacted them regarding zoonotic diseases. In contrast, $55.6 \%$ of the respondents mentioned that they had never contacted physicians regarding zoonoses. The findings of the present study were consistent with the findings of a study conducted among veterinarians $(n=370)$ in King County, as they have found that $>50 \%$ of the surveyed veterinarians had never spoken to physicians about zoonoses [22]. A study conducted in the USA revealed that $100 \%$ of physicians and $97 \%$ of veterinarians claimed to never or rarely contacted individuals from other professions when seeking advice on zoonotic disease risks or cases [21].

Veterinarians must also be aware of their responsibility to protect colleagues and associated staff, particularly nontechnical staff who may not know or be unaware of how to recognize and adequately protect themselves from zoonoses. A study conducted in Punjab state India has underscored that lack of awareness about zoonotic diseases among animal handlers compared to paraveterinarians and veterinarians places them at higher risk to contract Brucella organism [34]. In Australia, veterinarians are legally required to provide this information [24]. Zoonotic diseases pose risks of substantial liability to veterinary practice owners from both legal and occupational health perspectives in the USA [21]. The present study reported that $54.2 \%$ and 
$38.3 \%$ of the total respondents never and rarely discussed zoonoses among their client population and subordinates to educate or train them, respectively. The majority of King County veterinarians (77\%) agreed that it was very important to educate clients on zoonotic diseases prevention, but only $43 \%$ of the respondents-initiated discussions about zoonotic diseases prevention with clients on a daily basis, and $57 \%$ mentioned having client educational materials on zoonotic diseases in their practices [22].

Most of the respondents (87.7\%) showed an urgent need for the inclusion of OHS practices in continued veterinary education (CVE) programs. Although the adoption and implementation of specific infection control guidelines might increase work hours, it will be helpful to increase client trust and economic performance [21].

4.4. Awareness and Knowledge about Occupational Health and Safety (OHS). Approximately $34.9 \%$ and $47 \%$ of the veterinarians were aware of physical and biological hazards, whereas few (3.1\% and 3.6\%) were aware of chemical hazards and allergies, respectively, in their occupation. Similar results were observed among zoo veterinarians who were more likely to report inadequate knowledge of occupational hazards [4]. Surprisingly, knowledge of occupational hazards was inadequate among full-time American zoo veterinarians [45].

\section{Limitations of the Study}

The present study was conducted under a cross-sectional design and recorded the responses of practicing veterinarians through a set of questionnaires in a self-assessment manner. The information provided by the study subjects on past events can be a potential source of recall bias.

\section{Conclusions}

As per study observations, subjects were less attentive to their own health and lacked awareness and knowledge on OHS; this may pose a risk to Indian veterinarians for contracting the zoonotic disease(s). It is practically impossible to completely prevent exposure to biological hazards. However, education and training on zoonoses and implementation of effective ICPs can prevent biological hazards to a certain extent by identification of infected animals (for isolation), appropriate use of $\mathrm{PPE}$, proper handling and housing of animals, and personnel hygiene (such as proper handwashing) among veterinarians. To create awareness regarding prophylactic vaccination, veterinary medical schools should vaccinate veterinary students at the time of enrollment based on US veterinary school regulations. Guidelines in the "Guidebook on Adult Immunization in Occupational Health Settings (2020)" should be followed by working field veterinarians, and employers should adhere to these guidelines. Different countries have formulated and published infection control-related guidelines for veterinarians in their countries according to the field condition, such as the Compendium of Veterinary Standard, Precautions for Zoonotic Disease Prevention in Veterinary
Personnel, and National Association of State Public Health Veterinarians. Veterinary Infection Control Committee 2010 published in the Journal of American Veterinary Medical Association (JAVMA) as a guide for precautionary measures in America. The Australian Veterinary Association (AVA) has formulated and published a guide for the use of PPE; however, such comprehensive guidelines for Indian veterinarians are lacking. Moreover, routine health checkups, regular deworming of veterinarians, and medical record-keeping (including zoonoses screening) by the employer with monthly progress reports would help improve the quality of life of these professionals. The curricula in veterinary schools in the country should also emphasize occupational health and safety (OHS) practices. Standard protocols/guidelines for ICPs in veterinary clinics/dispensaries are lacking, and minimal emphasis is placed on measures to reduce the prevalence of zoonotic transmission, which is underscored by the lack of research published to date.

\section{Data Availability}

The [quantitative data] data used to support the findings of this study are included within the article.

\section{Conflicts of Interest}

The authors declare that there are no conflicts of interest regarding the publication of this article.

\section{Authors' Contributions}

RP planned, designed, conducted the survey, and prepared the manuscript. SD provided inputs in the design and conducted the data management and analysis. SB helped plan the study and revised the manuscript. All authors read and approved the final manuscript.

\section{Acknowledgments}

The authors are grateful to all the veterinarians who participated in this study and the Directorate of Animal Husbandry from both of the states of Gujarat and Maharashtra, India, for granting permission to conduct the study among veterinarians employed in their respective states. The support by technical staff (Jitendra Kumar and Ashvin Kumar Malhotra) while conducting the study is thankfully acknowledged.

\section{References}

[1] G. R. Pillai, Physical Injuries and Musculoskeletal Disorders (MSD) Among Veterinarians in Kerala: A Study on Occupational Risk Factors, Sree Chitra Tirunal Institute for Medical Sciences and Technology, Thiruvananthapuram, India, 2011.

[2] H. Kumar, K. Lokesha, C. Madhavaprasad, V. Shilpa, and N. Karabasanavar, "Occupational zoonoses in zoo and wildlife veterinarians in India,” Veterinary World, vol. 6, pp. 605-613, 2013.

[3] B. B. Chomel, "Zoonoses," Encyclopedia of Microbiology, pp. 820-829, 2009. 
[4] J. Jeyaretnam, H. Jones, and M. Phillips, "Disease and injury among veterinarians," Australian Veterinary Journal, vol. 78, pp. 625-629, 2000.

[5] S. Mishra and R. Palkhade, "Risk factors and prevalence of work-related injuries and accidents among veterinarians in India," Veterinary World, vol. 13, pp. 2555-2564, 2020.

[6] N. Travier, G. Gridley, A. Blair, M. Dosemeci, and P. Boffetta, "Cancer incidence among male Swedish veterinarians and other workers of the veterinary industry: a record-linkage study," Cancer Causes \& Control, vol. 14, pp. 587-593, 2003.

[7] K. T. Rim and C. H. Lim, "Biologically hazardous agents at work and efforts to protect workers' health: a review of recent reports," Safety and Health at Work, vol. 5, pp. 43-52, 2014.

[8] V. Khadayata and D. Aggarwal, "Knowledge, attitude, and practice about hygiene among livestock keepers in peri-urban area of Vadodara district, Gujarat," Indian Journal of Community Medicine, vol. 45, pp. 16-20, 2020.

[9] L. S. Taylor and M. Woolhouse, "Risk factors for human disease emergence," Philosophical transaction of Royal Society Biological Sciences, vol. 356, pp. 983-989, 2001.

[10] T. Epp and C. Waldner, "Occupational health hazards in veterinary medicine: zoonoses and other biological hazards," The Canadian Veterinary Journal $=L$ a revue Veterinaire Canadienne, vol. 53, pp. 144-150, 2012.

[11] A. Nienhaus, C. Skudlik, and A. Seidler, "Work-related accidents and occupational diseases in veterinarians and their staff," International Archives of Occupational and Environmental Health, vol. 78, pp. 230-238, 2005.

[12] J. S. Weese, A. S. Peregrine, and J. Armstrong, "Occupational health and safety in small animal veterinary practice: Part I-nonparasitic zoonotic diseases," The Canadian Veterinary Journal $=$ La revue Veterinaire Canadienne, vol. 43, pp. 631636, 2002.

[13] B. G. Mantur and S. K. Amarnath, "Brucellosis in India-a review," Journal of Biosciences, vol. 33, pp. 539-547, 2008.

[14] V. Proch, B. B. Singh, K. Schemann, J. P. S. Gill, M. P. Ward, and N. K. Dhand, "Risk factors for occupational Brucella infection in veterinary personnel in India," Transboundary and Emerging Diseases, vol. 65, pp. 791-798, 2018.

[15] R. Baer, W. Turnberg, D. Yu, and R. Wohrle, "Leptospirosis in a small animal veterinarian: reminder to follow standardized infection control procedures," Zoonoses and Public Health, vol. 57, pp. 281-284, 2010.

[16] M. M. de Rooij, B. Schimmer, B. Versteeg et al., "Risk factors of Coxiella burnetii ( $Q$ fever) seropositivity in veterinary medicine students," PLoS One, vol. 7, Article ID e32108, 2012.

[17] E. A. Whitney, R. F. Massung, A. J. Candee et al., "Seroepidemiologic and occupational risk survey for Coxiella burnetii antibodies among US veterinarians," Clinical Infectious Diseases, vol. 48, pp. 550-557, 2009.

[18] I. Habib and Z. Alshehhi, "Zoonotic disease management and infection control practices among veterinarians in the United Arab Emirates," Veterinary Sciences, vol. 8, p. 82, 2021.

[19] N. Sekar, N. K. Shah, S. S. Abbas, and M. Kakkar, "On behalf of the roadmap to combat zoonoses in India," PLoS One, vol. 6, Article ID e17120, 2011.

[20] J. E. Lessenger, "Disease and injury among veterinarians," in Agricultural Medicine: A Practical GuideSpringer, New York, NY, USA, 2006.

[21] J. G. Wright, S. Jung, R. C. Holman, N. N. Marano, and J. H. McQuiston, "Infection control practices and zoonotic disease risks among veterinarians in the United States," Journal of the American Veterinary Medical Association, vol. 232, pp. 1863-1872, 2008.
[22] B. A. Lipton, S. G. Hopkins, J. E. Koehler, and R. F. DiGiacomo, "A survey of veterinarian involvement in zoonotic disease prevention practices," Journal of the American Veterinary Medical Association, vol. 233, pp. 1242-1249, 2008.

[23] M. Thrusfield, Veterinary Epidemiology, p. 626, 3rd edition, Blackwell Publsihing, Oxford, UK, 2009.

[24] J. Jeyaretnam and H. Jones, "Physical, chemical and biological hazards in veterinary practice," Australian Veterinary Journal, vol. 78, pp. 751-758, 2000.

[25] M. Chigerwe, L. Barter, J. E. Dechant, J. D. Dear, and K. A. Boudreaux, "A preliminary study on assessment of wellbeing among veterinary medical house officers," PLoS One, vol. 16, Article ID e0253111, 2021.

[26] R. Shome, T. Kalleshamurthy, P. B. Shankaranarayana et al., "Prevalence and risk factors of brucellosis among veterinary health care professionals," Pathogens and Global Health, vol. 111, pp. 234-239, 2017.

[27] B. F. Kingscote, "Leptospirosis: an occupational hazard to veterinarians," Canadian Veterinary Journal, vol. 27, pp. 7881, 1986.

[28] S. Shuhaiber, G. Koren, R. Boskovic, T. R. Einarson, O. P. Soldin, and A. Einarson, "Seroprevalence of Toxoplasma gondii infection among veterinary staff in Ontario, Canada (2002): implications for teratogenic risk," BMC Infectious Diseases, vol. 3, p. 8, 2003.

[29] R. M. Moore Jr., Y. M. Davis, and R. G. Kaczmarek, “An overview of occupational hazards among veterinarians, with particular reference to pregnant women," American Industrial Hygiene Association Journal, vol. 54, pp. 113-120, 1993.

[30] P. S. M. Wiggins, R. Green, and S. Samuels, "Prevalence of hazardous exposures in veterinary practice," American Journal of Industrial Medicine, vol. 16, 1989.

[31] K. Reijula, K. Rasanen, M. Hamalainen et al., "Work environment and occupational health of Finnish veterinarians," American Journal of Industrial Medicine, vol. 44, pp. 46-57, 2003.

[32] A. L. Hafer, R. L. Langley, and W. E. M. Morrow, "Occupational hazards reported by swine veterinarians in the United States," JSHAP, vol. 4, pp. 128-141, 1996.

[33] B. Awosile and E. Omoshaba, "Hazards exposures of workers of animal related occupations in Abeokuta southwestern, Nigeria," Journal of Veterinary Advances, vol. 3, no. 1, pp. 9-19, 2013.

[34] H. K. Tiwari, V. Proch, B. B. Singh et al., "Brucellosis in India: comparing exposure amongst veterinarians, para-veterinarians and animal handlers," One Health, vol. 14, Article ID 100367, 2022.

[35] K. Dowd, M. Taylor, J.-A. L. M. L. Toribio, C. Hooker, and N. K. Dhand, "Zoonotic disease risk perceptions and infection control practices of Australian veterinarians: call for change in work culture," Preventive Veterinary Medicine, vol. 111, pp. 17-24, 2013.

[36] I. A. Odetokun, A. T. Jagun-Jubril, B. A. Onoja, Y. S. Wungak, I. A. Raufu, and J. C. Chen, "Status of laboratory biosafety and biosecurity in veterinary research facilities in Nigeria," Saf Health Work, vol. 8, pp. 49-58, 2017.

[37] M. K. Sudarshan, S. N. Madhusudana, B. J. Mahendra et al., "Assessing the burden of human rabies in India: results of a national multi-center epidemiological survey," International Journal of Infectious Diseases, vol. 11, pp. 29-35, 2007.

[38] P. Nigam and A. Srivastav, "Proscenia opasnosti pri radu specijalista za zastitu zdravlja divljih zivotinja u Indiji," Veterinarski Arhiv, vol. 81, pp. 731-741, 2011. 
[39] P. A. Koul, S. Swaminathan, T. Rajgopal et al., "Adult immunization in occupational settings: a consensus of Indian experts," Indian Journal of Occupational and Environmental Medicine, vol. 24, pp. 3-15, 2020.

[40] J. Jackson and A. Villarroel, "A survey of the risk of zoonoses for veterinarians," Zoonoses and public health, vol. 59, pp. 193-201, 2012.

[41] F. M. Tambuwal, A. Shittu, M. i. B. Abubakar et al., "A survey of veterinary hospitals in Nigeria for the presence of some bacterial organisms of nosocomial and zoonotic potential," Veterinaria Italiana, vol. 45, pp. 235-241, 2009.

[42] A. Moodley, E. C. Nightingale, M. Stegger, S. S. Nielsen, R. L. Skov, and L. Guardabassi, "High risk for nasal carriage of methicillin-resistant Staphylococcus aureus among Danish veterinary practitioners," Scandinavian Journal of Work, Environment \& Health, vol. 34, pp. 151-157, 2008.

[43] E. A. Jenner, B. Fletcher, P. Watson, F. A. Jones, L. Miller, and G. M. Scott, "Discrepancy between self-reported and observed hand hygiene behaviour in healthcare professionals," Journal of Hospital Infection, vol. 63, pp. 418-422, 2006.

[44] C. Fuller, J. Savage, S. Besser et al., "“The dirty hand in the latex glove": a study of hand hygiene compliance when gloves are worn," Infection Control and Hospital Epidemiology, vol. 32, pp. 1194-1199, 2011.

[45] D. J. Hill, R. L. Langley, and W. M. Morrow, "Occupational injuries and illnesses reported by zoo veterinarians in the United States," Journal of Zoo and Wildlife Medicine, vol. 29, pp. 371-385, 1998. 